\title{
Urine drug screening for early detection of unwitting use of fentanyl and its analogues among people who inject heroin in Sydney, Australia
}

Barratt, Monica; Latimer, Julie; Jauncey, Marianne; Tay, Emma; Nielsen, Suzanne

https://researchrepository.rmit.edu.au/esploro/outputs/9921861194701341/filesAndLinks?institution=61RMIT_INST\&index=null

Barratt, M., Latimer, J., Jauncey, M., Tay, E., \& Nielsen, S. (2018). Urine drug screening for early detection of unwitting use of fentanyl and its analogues among people who inject heroin in Sydney, Australia. Drug and Alcohol Review, 37(7), 847-850. https://doi.org/10.1111/dar.12864

Document Version: Accepted Manuscript

Published Version: https://doi.org/10.1111/dar.12864

Repository homepage: https://researchrepository.rmit.edu.au

(c) 2018 Australasian Professional Society on Alcohol and other Drugs

Downloaded On 2023/04/26 17:55:42 +1000 
Urine drug screening for early detection of unwitting use of fentanyl and its analogues among people who inject heroin in Sydney, Australia

RUNNING TITLE: Fentanyl-laced heroin in Australia

Monica J. Barratt, PhD, Research Fellow, Drug Policy Modelling Program, National Drug and Alcohol Research Centre, UNSW Sydney, Australia; National Drug Research Institute, Faculty of Health Sciences, Curtin University, Perth, Australia; Behaviours and Health Risks Program, Burnet Institute, Melbourne, Australia.

Julie Latimer, BN (Hons), Nursing Unit Manager, Uniting Medically Supervised Injecting Centre, Sydney, Australia.

Marianne Jauncey, BMed, MPH(Hons), FAFPHM, Director, Uniting Medically Supervised Injecting Centre, Sydney, Australia.

Emma Tay, BMed, Clinical Pharmacology \& Addiction Medicine Advanced Trainee, St Vincent's Hospital, Sydney, Australia.

Suzanne Nielsen, Associate Professor, Monash Addiction Research Centre, Melbourne, Australia; National Drug and Alcohol Research Centre, UNSW Sydney, Australia.

Corresponding author:

Suzanne Nielsen

Monash University, Peninsula Campus

Monash Addiction Research Centre

Building G 3rd floor, McMahons Road

Frankston VIC 3199, Australia

T: +61 399044641

Suzanne.Nielsen@monash.edu 


\begin{abstract}
INTRODUCTION AND AIMS: North America has witnessed a dramatic rise in fatal opioid overdoses due to the unwitting consumption of non-pharmaceutical fentanyl and its analogues. While some of the drivers of this crisis — including profitability and access to high-potency opioids through internet sources - also apply in Australia, to our knowledge, there have been no ongoing surveillance studies of local populations. Therefore, this pilot study aimed to detect unintentional fentanyl consumption among people who inject heroin through instant urine screening, and determine the feasibility and acceptability of voluntary urinalysis of clients at the Medically Supervised Injecting Centre (MSIC), Kings Cross, Sydney. DESIGN AND METHODS: Brief surveys and urine drug screens were conducted with 67 participants in Wave 1 (October 2017) and 51 participants in Wave 2 (March 2018). Urine samples were tested with BTNX Rapid Response ${ }^{\mathrm{TM}}$ fentanyl urine strip test at a detection level of $20 \mathrm{ng} / \mathrm{ml}$ norfentanyl. These strips also cross-react to numerous fentanyl analogues. RESULTS: There were no cases where positive urine tests suggested unwitting fentanyl use detected in this study. DISCUSSION AND CONCLUSIONS: These negative findings contrast sharply with similar Canadian studies. While no cases of fentanyl-laced heroin use have been detected so far, we have demonstrated that this surveillance design is low-cost, feasible and scalable approach to monitoring the considerable public-health threat of undetected fentanyl and its analogues in Australia. Further validation of cross-reactivity of test strips would strengthen this method.
\end{abstract}

Keywords: Fentanyl, Heroin, Designer Drugs, Sentinel Surveillance, Immunoassay (up to 5, from MeSH only)

Submission type: Brief Report

Word count abstract $=236$ (Maximum of 250)

Word count body of paper $=1498$ (Maximum of 1500)

Number of references $=20$ (Maximum of 20)

Number of tables/figures $=2$ (Maximum of 2$)$ 


\section{Introduction}

In North America, high-potency illicit synthetic opioids such as fentanyl and its analogues (e.g., acetylfentanyl, furanylfentanyl, carfentanil, U-47700 and MT-45), have caused a critical public health problem due to the dramatic increase in fatal overdoses $[1,2]$. Greater mortality is attributed to their higher potency. They are also often sold as 'heroin' [3] therefore consumers may be unaware of the increased potency and need for dose adjustments. Higher than usual doses of naloxone, the opioid antidote, may be required for overdoses involving these substances [1, 2]. A key driver of the crisis in North America is the increased profits for manufacturers and/or suppliers following the addition of high-potency opioids to powder sold as 'heroin' to increase its potency [4, 5].

In Australia, diverted pharmaceutical fentanyl has been associated with increased risk of overdose [6]. Non-pharmaceutical fentanyl and analogues are also available through cryptomarkets or darknet markets [7]. Given that at least some of the drivers of the crisis in North America also apply in Australia (including low cost access to potent new drugs and accessibility of cryptomarkets) it is important that Australia positions itself to rapidly detect and respond if evidence of illicit highpotency opioids in the heroin supply emerges locally [8].

There is some evidence of an Australian threat. A cluster of 9 deaths in Melbourne was identified that resulted from a combination of heroin and fentanyl derivatives ('fentanyl-laced heroin') [9]. The Australian Border Force (ABF) have also stopped shipments of carfentanil [10]. A 2017 report described regular ABF intercepted shipments of illicit opioids, "sometimes even three times a day" [11]. Despite these signs, there have been no ongoing surveillance studies of affected populations in Australia to our knowledge.

Urine screening for fentanyl and derivatives is a relatively easy way of incorporating surveillance within client services. Four Canadian studies [12-15] have utilised urine screening in community settings to monitor the unintended consumption of fentanyl (and analogues). In 2015, Amlani et al. [14] recruited 242 clients who reported illicit drug use. Of the $29 \%(n=70)$ whose urine tested positive for fentanyl and analogues, 73\% were using fentanyl unknowingly. Hayashi et al. (2016) [13] tested 
the urine of 452 people who reported injecting drugs, with $20 \%(n=89)$ testing positive for fentanyl and analogues. Of these $89,85 \%$ were consuming fentanyl unknowingly. Jones et al. (2017) [12] collected 5 monthly urine from 237 participants, including individuals who reported nonprescribed opioid use and/or the use of opiate substitution treatments. Nearly all (83 of 91; 91\%) participants who reported nonprescribed opioid use (including heroin) submitted at least 1 fentanyl-positive urine sample; $82 \%$ of these were using fentanyl unknowingly. A pilot study by Mema et al. (2017) with 24 rural harm-reduction clients, found 4 tested positive for fentanyl, but most of these were not surprised by the result, perhaps due to the acceptance of fentanyl adulteration in Canadian heroin supplies [15]. All studies used BTNX Rapid Response ${ }^{\mathrm{TM}}$ fentanyl urine strips: Amlani et al. and Mema et al. used the fentanyl-only strip, Hayashi et al. used the multi-panel screen to detect fentanyl and 8 other substances, and Jones et al. used fentanyl, opiates and methadone strips. The success of this surveillance method in these Canadian studies inspired our own study repeating similar methodology in Australia.

\section{$\operatorname{Aim}$}

This pilot study aimed to: (a) detect unintentional fentanyl consumption among people who inject heroin through instant urine screening, and (b) determine the feasibility and acceptability of voluntary urinalysis for participants at the Medically Supervised Injecting Centre (MSIC), Kings Cross, Sydney.

\section{Method}

Brief surveys and urine drug screens were conducted 118 times with 104 clients of the MSIC: 67 participants in Wave 1 (October 2017) and 51 participants in Wave 2 (March 2018), with 14 clients participating in both waves. Clients were eligible if they reported heroin use within the past 48 hours and were $18+$ years old. Clients were deemed ineligible if they: had already participated in that data collection wave, lacked sufficient comprehension of English to provide informed consent, or were assessed as either intoxicated or as experiencing significant psychological distress or other psychiatric symptoms, though no participants were excluded for the latter reason. Eligible clients were approached by staff following their drug injection at MSIC. Ethics approval was obtained from 
UNSW Sydney (\#HC17283). Materials were distributed to MSIC's consumer group to check for suitability and acceptability of study design, given that urine testing may be perceived as threatening if anonymity is not preserved.

Participants completed a brief survey, covering demographics, recent drug use (covering the current day, and the two days prior reflecting the approximately 48-hour detection period for opioids with urine drug screens), and more detailed questions on heroin and fentanyl use. At survey completion, clients provided a urine sample which was tested by an MSIC staff member with the Rapid Response $^{\mathrm{TM}}$ Single Drug Test Strip (Fentanyl) (BTNX) [16]. Samples were tested for fentanyl/norfentanyl (cut-off concentration $20 \mathrm{ng} / \mathrm{mL}$, with reported cross-reactivities to 3methylfentanyl, acetylfentanyl, butyrylfentanyl, carfentanil, furanylfentanyl, ocfentanil, pfluorofentanyl, remifentanil, sufentanil and valeryl fentanyl). After 5 minutes, results were provided to the clients. Where the test was positive for fentanyl, the client was offered further information and counselling regarding risks and harm reduction advice regarding the consumption of high-potency opioids, in line with existing clinical governance of MSIC. Urine samples were disposed of immediately and the clients' confidentiality was maintained. Participants entered a draw for a $\$ 100$ gift voucher. Aggregate results were displayed to clients following closure of each wave.

\section{Results}

\section{Demographics}

Of the 104 participants, $73 \%$ identified as male, $25 \%$ as female, and $2 \%$ as transgender. Their median age was 45 years (IQR 40-53; range 23-65).

\section{Self-reported heroin use and supply characteristics}

Self-reported heroin use and supply characteristics are detailed in Table 1. Figure 1 presents selfreported drug types used in the past 3 days.

[Insert Figure 1 and Table 1 about here] 


\section{Fentanyl detection}

In Wave 1, 3 participants reported use of fentanyl patches and 2 of these tested positive for fentanyl on the urine screen. The other participant reported fentanyl use only on 'the day before yesterday', suggesting levels were likely to be below detectable concentrations at the time of testing. The other 64 participants who did not report fentanyl use did not test positive on the fentanyl urine screen. In Wave 2, 1 participant self-reported fentanyl patch use, consistent with their positive urine result. The remaining 50 participants did not report fentanyl use and did not test positive for fentanyl via the urine screen. Thus, no cases of fentanyl-laced heroin were detected across either wave of the study.

\section{Discussion}

Unlike similar Canadian studies [12-15], we found no evidence of fentanyl-laced heroin through urine screens among people who inject 'heroin' in Sydney, Australia. Regarding feasibility and acceptability, clients appeared interested in whether their heroin contained fentanyl, as demonstrated among similar populations elsewhere $[15,17,18]$. The instant urine tests were easy to use in a community health setting. Some clients found it difficult to provide a sample, possibly due to effects of recent heroin consumption. Notwithstanding, we demonstrated that this is an overall low-cost, feasible and scalable approach to monitor the considerable public-health threat of undetected fentanyl in the Sydney heroin market.

\section{Limitations}

Laboratory analysis of urine samples by chromatography and mass spectrometry would allow more sensitive and specific identification of a wider range of fentanyl analogues, in addition to validating results seen in urine screens that employ immunoassay analysis. The wide cross-reactivity of the BTNX Rapid Response ${ }^{\mathrm{TM}}$ urine test strip to a range of fentanyl analogues was considered beneficial in this context, although external validation of the accuracy of reported cross-reactivities is required. Not all fentanyl analogues that have been associated with hospitalisations and fatalities [19] are detectable by these test strips, which may present the risk of falsely reassuring clients. The timing of last reported heroin (and fentanyl) use relative to the timing of urine testing is important to note, as 
detectable concentrations can diminish over time. Another option for future surveillance studies of the heroin market is on-site drug sample testing using field-ready portable gas chromatography-mass spectrometry (GC-MS) equipment [20]. Additionally, Fourier-transform infrared spectroscopy (FTIR), Raman spectroscopy and BTNX urine test strips (used off-label) have all been used to detect fentanyl in drug samples [17]. A combination of these technologies would ideally be used to validate each testing method against the GC-MS gold standard.

Further work should establish the extent to which positive tests result in changes to substance use patterns. This is a promising area of inquiry: previous studies have found that point-of-care detection of fentanyl and analogues in drug samples ('drug checking') offers clients an opportunity to avoid consuming specific drug batches [17, 18] and increases reports of harm-reducing practices [15].

\section{Conclusions}

We have reported initial findings from an ongoing surveillance study of fentanyl adulteration in heroin markets accessed by Sydney's MSIC clients. So far, no signs of unwitting use of fentanyl have been detected. Ongoing monitoring will ensure that any changes to this situation will be identified.

\section{Acknowledgements}

We thank all the MSIC clients who gave up their time to participate in this study. Thanks to Michael Gilbert for initial advice when setting up this study and Michala Kowalski for assistance with data entry. MB and SN are supported by fellowships from the National Health and Medical Research Council (APP1070140 \& APP1132433). The National Drug and Alcohol Research Centre and the National Drug Research Institute are supported by funding from the Australian Government under the Drug and Alcohol Program Fund. We also acknowledge the contribution of the Victorian Operational Infrastructure Support Program received by the Burnet Institute. The content is solely the responsibility of the authors and does not necessarily represent the official views of the funders listed. An earlier version of this paper was presented at the APSAD Scientific Alcohol and Drug Conference, 12-15 November 2017, Melbourne. 


\section{Declaration of interests}

$\mathrm{SN}$ is an investigator on an untied educational grant from Indivior and her institution has received honoraria and had travel costs covered to provide training on identifying and treating codeine dependence by Indivior. There are no other relevant interests to declare. 


\section{References}

1. Suzuki J, El-Haddad S. A review: Fentanyl and non-pharmaceutical fentanyls. Drug Alcohol Depend. 2017;171:107-16.

2. Prekupec MP, Mansky PA, Baumann MH. Misuse of Novel Synthetic Opioids: A Deadly New Trend. J Addict Med. 2017;11(4):256-65.

3. Ciccarone D, Ondocsin J, Mars SG. Heroin uncertainties: Exploring users' perceptions of fentanyl-adulterated and -substituted 'heroin'. International Journal of Drug Policy. 2017;46:146-55.

4. Peterson AB, Gladden RM, Delcher C, Spies E, Garcia-Williams A, Wang Y, et al. Increases in Fentanyl-Related Overdose Deaths - Florida and Ohio, 2013-2015. MMWR Morb Mortal Wkly Rep. 2016;65(33):844-9.

5. Gladden RM, Martinez P, Seth P. Fentanyl Law Enforcement Submissions and Increases in Synthetic Opioid-Involved Overdose Deaths - 27 States, 2013-2014. MMWR Morb Mortal Wkly Rep. 2016;65(33):837-43.

6. Latimer J, Ling S, Flaherty I, Jauncey M, Salmon AM. Risk of fentanyl overdose among clients of the Sydney Medically Supervised Injecting Centre. Int J Drug Policy. 2016;37:111-4.

7. Quintana P, Ventura M, Grifell M, Palma A, Galindo L, Fornís I, et al. The hidden web and the fentanyl problem: Detection of ocfentanil as an adulterant in heroin. International Journal of Drug Policy. 2017;40:78-83.

8. McKeown HE, Rook TJ, Pearson JR, Jones OAH. Is Australia ready for fentanyl? Science \& Justice. 2018.

9. Rodda LN, Pilgrim JL, Di Rago M, Crump K, Gerostamoulos D, Drummer OH. A Cluster of Fentanyl-Laced Heroin Deaths in 2015 in Melbourne, Australia. Journal of Analytic Toxicology. 2017;41(4):318-24.

10. Burdon D. Warning for Canberra's drug users this summer after deadly opiate made it to Australia Canberra Times. 2016 December 21.

11. Miranda C. Rise in dark web drugs sparks fears in Australia of an likely epidemic. Herald Sun. 20179 April.

12. Jones AA, Jang K, Panenka WJ, Barr AM, MacEwan GW, Thornton AE, et al. Rapid change in fentanyl prevalence in a community-based, high-risk sample. JAMA Psychiatry. 2018;75(3):298300 .

13. Hayashi K, Milloy MJ, Lysyshyn M, DeBeck K, Nosova E, Wood E, et al. Substance use patterns associated with recent exposure to fentanyl among people who inject drugs in Vancouver, Canada: A cross-sectional urine toxicology screening study. Drug \& Alcohol Dependence.

2018;183:1-6.

14. Amlani A, McKee G, Khamis N, Raghukumar G, Tsang E, Buxton JA. Why the FUSS

(Fentanyl Urine Screen Study)? A cross-sectional survey to characterize an emerging threat to people who use drugs in British Columbia, Canada. Harm Reduct J. 2015;12:54.

15. Mema SC, Sage C, Popoff S, Bridgeman J, Taylor D, Corneil T. Expanding harm reduction to include fentanyl urine testing: results from a pilot in rural British Columbia. Harm Reduction Journal. 2018;15(1):19.

16. BTNX Inc. Rapid response: fentanyl (FYL) test strip (urine) product insert. Markham, ON, Canada; 2016.

17. Johns Hopkins Bloomberg School of Public Health. Fentanyl Overdose Reduction Checking Analysis Study (FORECAST). Summary Report. Baltimore, MA: Bloomberg School of Public Health, Johns Hopkins; 2018.

18. Kennedy MC, Scheim A, Rachlis B, Mitra S, Bardwell G, Rourke S, et al. Willingness to use drug checking within future supervised injection services among people who inject drugs in a midsized Canadian city. Drug Alcohol Depend. 2018.

19. Drummer OH. Fatalities caused by novel opioids: a review. Forensic Sciences Research. 2018:1-16.

20. Sisco E, Verkouteren J, Staymates J, Lawrence J. Rapid detection of fentanyl, fentanyl analogues, and opioids for on-site or laboratory based drug seizure screening using thermal desorption DART-MS and ion mobility spectrometry. Forensic Chemistry. 2017;4(Supplement C):108-15. 
Table 1. Self-reported heroin use and supply characteristics

\begin{tabular}{|c|c|c|}
\hline & Wave 1 (Oct 2017) & Wave 2 (Mar 2018) \\
\hline & $\mathrm{N}=67$ & $\mathrm{~N}=51$ \\
\hline \multicolumn{3}{|l|}{ Heroin use $(\%)^{1}$} \\
\hline All three days & 46 & 55 \\
\hline Two of three days & 24 & 24 \\
\hline One of three days & 30 & 22 \\
\hline Number of heroin injections ${ }^{1}$ & 4 (IQR 2-6; R 1-24) & 4 (IQR 3-9; R 1-60) \\
\hline Number of heroin sources ${ }^{1}$ & 2 (IQR 1-2; R 1-7) & 1 (IQR 1-2; R 1-20) \\
\hline Sourced heroin locally ${ }^{2}(\%)$ & 83 & 83 \\
\hline Sourced heroin elsewhere ${ }^{2}(\%)$ & 26 & 33 \\
\hline \multicolumn{3}{|c|}{ Perceived heroin supply changes (\%) } \\
\hline Nothing unusual & 48 & 70 \\
\hline Heroin more potent than usual & 28 & 9 \\
\hline Heroin less potent than usual & 14 & 11 \\
\hline Other heroin supply changes & 10 & 10 \\
\hline
\end{tabular}

${ }^{1}$ Time period: today, yesterday and the day before yesterday. Reporting median, interquartile range (IQR) and range (R). ${ }^{2}$ Multiple response. 
Figure 1. Self-reported drug types used today, yesterday and the day before yesterday (\%)

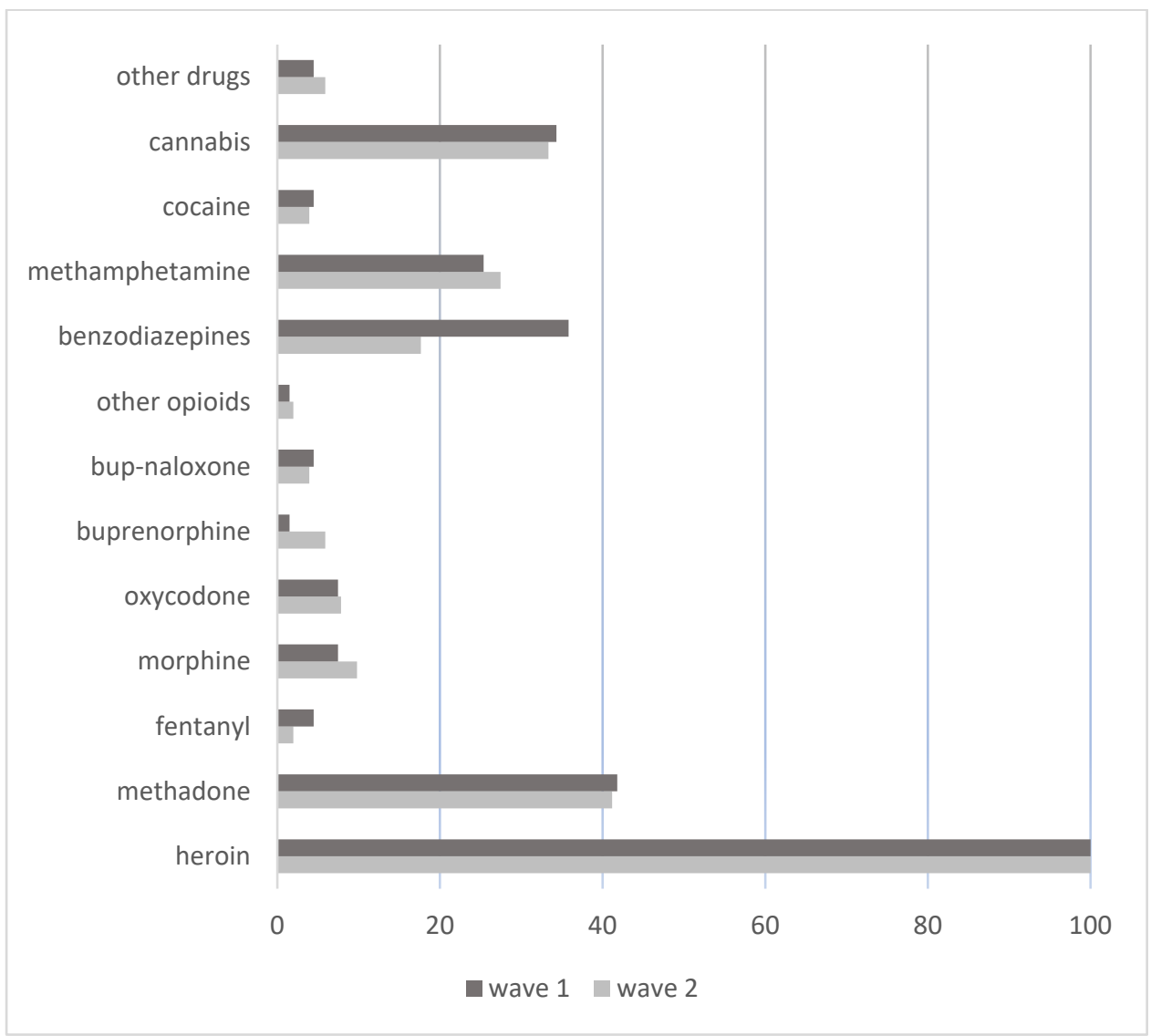

